\title{
QED blue-sheet effects inside black holes
}

\author{
Lior M. Burko \\ Department of Physics, Technion-Israel Institute of Technology, 32000 Haifa, Israel.
}

(August 26, 2018)

The interaction of the unboundedly blue-shifted photons of the cosmic microwave background radiation with a physical object falling towards the inner horizon of a Reissner-Nordström black hole is analyzed. To evaluate this interaction we consider the QED effects up to the second order in the perturbation expansion. We then extrapolate the QED effects up to a cutoff, which we introduce at the Planckian level. (Our results are not sensitive to the cutoff energy.) We find that the energy absorbed by an infalling observer is finite, and for typical parameters would not lead to a catastrophic heating. However, this interaction would almost certainly be fatal for a human being, or other living organism of similar size. On the other hand, we find that smaller objects may survive the interaction. Our results do not provide support to the idea that the Cauchy horizon is to be regarded as the boundary of spacetime.

PACS numbers: 04.40.Nr, 04.70.Bw 


\section{INTRODUCTION}

The question whether there is a boundary to spacetime is of fundamental interest for a more complete understanding of the structure of spacetime and spacetime singularities. It has been argued [1] that the inner horizon of black holes (BHs) may be such a boundary. The inner horizon is a 3-surface of infinite blue shift. Namely, any ingoing radiation, even very mild and well-behaved in the external universe, is infinitely blue-shifted at the inner horizon [2], also known as the Cauchy horizon (CH).

Even if spacetime continues beyond the $\mathrm{CH}$, it is still not clear whether (extended) physical objects can traverse it without being destroyed by the infinite concentration of energy density and by the curvature singularity expected to lurk there. The main goal of this Paper is to study the following question within some (very) simplified model: Are extended objects completely destroyed by the infinite concentration of energy density expected to exist at the $\mathrm{CH}$ ? To address this question we shall deal only with the direct electrodynamic effects of the blue sheet. Thus, we ignore the tidal effects on the infalling object. (These effects were found to be negligible at the early parts of the inner horizon singularity - see Refs. 3,4.4.) Also, we ignore the influence of local curvature on the QED processes. Namely, we assume that the size of the object is smaller than the typical radius of spacetime curvature near the $\mathrm{CH}$. However, there always exist gravitational perturbations which produce a diverging curvature at the $\mathrm{CH}$, which means that the radius of curvature vanishes there. Our assumption is valid only if - for the sake of evaluating the QED effects - we ignore the gravitational perturbations. The modification of the interaction by the metric perturbations is obviously a non-linear effect, as it is quadratic in the perturbation's amplitude. This non-linear effect remains the subject of future research. We do not expect, however, this non-linear effect to significantly alter the linear-order interaction. The gravitational analogue of this problemthe object's interaction with the divergent tidal forcesdemonstrates this reasoning: As implied from the analysis of Ref. [4 on the strength of the $\mathrm{CH}$ singularity in spinning $\mathrm{BHs}$ - where it has been demonstrated that the non-linear gravitational interaction with an object may be negligible - we do not expect higher-order contributions to change the general picture significantly. Thus, in this work we restrict ourselves to linear effects only, and take the background to be unperturbed.

It is believed that when an astrophysical $\mathrm{BH}$ is formed (through a gravitational collapse process), after perturbations die off a Kerr BH is left. It turns out that mathematical analysis of perturbations in the Kerr BH is very complicated, due to the lack of spherical symmetry of the background [5]. The Reissner-Nordström (RN) BH is therefore often used as a toy model for the more physical Kerr BH. This model can be justified by the similarity between the inner causal structures of the two solutions and by similar blue-shift effects.

In principle, any attempt to predict the fate of an object which tries to cross the $\mathrm{CH}$ is limited due to the ambiguity in the evolution of the various physical fields beyond the $\mathrm{CH}$. It therefore makes sense to restrict attention to the object's history up to the CH. (Obviously, a non-catastrophic approach to the $\mathrm{CH}$ is a necessary condition for a peaceful crossing of the latter.) This is the approach we shall take here.

There are two generic sources of electromagnetic radiation which irradiate the $\mathrm{CH}$. First, any realistic $\mathrm{BH}$ is surrounded by the 'tails' of radiation - which result from the backscattering off the curvature of spacetime of the electromagnetic waves created by the evolution of (electromagnetic) multipole moments in the star during the collapse. These 'tails' decay at the event horizon according to an inverse power law in advanced time [6, (7). Second, any BH constantly captures photons which originate from the relic cosmic microwave background radiation (CBR). It turns out that both the flux and the frequency of these photons are infinitely blue-shifted at the $\mathrm{CH}$. In this Paper we shall focus on this second source - the CBR photons.

Linear analyses [8] have shown that perturbations which outside the $\mathrm{BH}$ decay according to an inversepower law in external time, diverge exponentially at the $\mathrm{CH}$. Also, the projection of the energy-momentum tensor on the worldline of an infalling observer diverged at the $\mathrm{CH}$ [9]. Burko and Ori (BO) [10] have recently shown that this divergence does not necessarily mean the complete burning up of physical objects on their attempt to traverse the $\mathrm{CH}$. However, BO treated the electromagnetic field as a classical Maxwell field, and did not consider possible high-energy QED processes. They also did not discuss the other generic radiation source, namely the infalling photons of the CBR. Cosmological effects were studied by Balbinot et al [11] who considered the traversability of a wormhole consisting of closed Friedmann-Robertson-Walker universes connected by a RN BH. They showed that the $\mathrm{CH}$ singularity was classically mild, but did not consider, however, the direct electrodynamic interaction of the CBR photons with the infalling object.

In this Paper we analyze the (ultra-relativistic) electrodynamic interaction of the blue-shifted CBR photons with an infalling object. We consider the interaction up to second order in QED perturbation theory, and find that this interaction is dominated by the production of electron-positron pairs in the field of the atomic electrons and the nuclei of the matter comprising the object, and by the ionization of the matter due to the photo-electric and Compton effects. (We have not looked at the effects of higher-order interactions.) We shall show, that if the infalling object is small compared to the typical penetration length of the electrodynamic processes (i.e., if the typical length of the object in the radial direction is of order $1-10 \mathrm{~cm}$ or smaller), only a small fraction of its atoms will interact with the infalling photons. 
As the energy of the incident photons diverges on the $\mathrm{CH}$, the photon's energy becomes super-Planckian at some point. As physics at super-Planckian energies is as yet completely unknown, we introduce a cutoff when the photon's energy becomes Planckian. Moreover, physics is as yet unknown even at lower energies. We (quite artificially) face this difficulty by extrapolating the QED cross sections up to a cutoff introduced at the Planck energy. While this is clearly a very simplified model for the interaction at high energies, we hope that it may yield a reasonable order-of-magnitude estimate for the physical effects. This estimate may be reasonable if higher order QED effects and higher-energy interactions do not change our conclusions drastically. We also do not consider here semi-classical effects, like those studied in Refs. 12 14].

We shall show that up to Planck energy the actual heating of the infalling matter will be bounded and for typical astrophysical parameters could be even quite small. Also, this heating is not sensitive to the cutoff energy scale. For a more complete understanding, one should use electroweak and GUT cross sections at corresponding energies, and consider QED interactions of higher order in perturbation theory, as well as the formation of more massive pairs and the back-reaction on the geometry. Nevertheless, we believe that our analysis can yield a vague order-of-magnitude estimate for the actual interaction strength. Finally, we note that as the interaction strength we calculate is bounded and is not drastically large, we do not find support to the hypothesis that there is no physical continuation of the geometry beyond the $\mathrm{CH}$.

The organization of this Paper is as follows: In Section II we evaluate the number of the incident photons. Then, in Section III, we assess the actual number of the photons interacting with the infalling object, and in Section IV we study the effect of these interaction on the object.

\section{THE NUMBER OF INCIDENT PHOTONS}

Let us consider an isolated $\mathrm{RN} \mathrm{BH}$ with mass $M$ and electric charge $Q_{*}$. We assume that this $\mathrm{BH}$ is surrounded by the CBR photons, which away from the $\mathrm{BH}$, are distributed homogeneously and isotropically, according to the standard Big Bang cosmological model. We also assume that the cosmology is described by the standard Robertson-Walker model with critical density parameter $(\Omega=1)$. (In fact, we find that our results are insensitive to the details of cosmological model.)

The flux of photons which fall into the $\mathrm{BH}$ is $d n(t) / d t=\sigma_{\text {cap }} \rho(t) c$, where $\sigma_{\text {cap }}$ is the cross section for capture of photons by the BH. This cross section is a function of $Q_{*}$ and $M$, and is given explicitly in Ref. [15]. $\rho(t)$ is the number density of the CBR photons at the external time $t$ away from the $\mathrm{BH}$, and $c$ is the speed of light. We make the simplifying assumption that the infalling photons move along radial trajectories described by geometrical optics. (This approximation seems to be justified due to the divergent blue-shift.) When the object gets close to the $\mathrm{CH}$, the flux of incident photons (per unit proper time) is the (external time) absorption rate, $d n / d t$, multiplied by two geometric factors: First, by $S /\left(4 \pi r_{-}^{2}\right)$, where $S$ is the object's cross sectional area (in the $\theta-\phi$ plane), and $r_{-}=M-\left(M^{2}+Q_{*}^{2}\right)^{1 / 2}$ is the inner horizon. (We take here $c=1$. We also take Newton's constant $G=1$ throughout.) This is the fraction of the solid angle $4 \pi$ from which photons can hit the object. Second, the factor $d t / d \tau$, which relates the external time $t$ to the observer's proper time $\tau$. Here, the time $t$ can be taken along a line of constant $r=r_{0} \gg M .\left[r_{0}\right.$ is much smaller than the typical cosmological radius of curvature. The relation between $t$ (at $r=r_{0}$ ) and $\tau$ (at the object's world line) is determined by ingoing null geodesics. Here, $r$ is the radial Schwarzschild coordinate.] A straightforward calculation yields $d t / d \tau \approx\left(\kappa_{-} \tau\right)^{-1}$, where $\kappa_{-}$is the surface gravity of the $\mathrm{CH}$ defined by $\kappa_{-} \equiv \frac{1}{2}|d f / d r|_{r=r_{-}}$. Here, $f=1-2 M / r+Q_{*}^{2} / r^{2}$. (This approximation for $d t / d \tau$ is valid near the $\mathrm{CH}$ to leading order in $r-r_{-}$.) We set $\tau$ such that $\tau=0$ is the value of $\tau$ corresponding to the observer's arrival at the $\mathrm{CH}$. Therefore, the flux of incident photons is $\dot{n}(\tau) \equiv d n / d \tau=\rho(t) \sigma_{\text {cap }} S /\left(4 \pi r_{-}^{2}\right)\left|\kappa_{-} \tau\right|^{-1}$. Now, the variation of $\rho$ with $t$, due to the cosmological evolution, is unimportant to our problem: The relevant interval of $t$ (up to the Planckian cutoff) is negligible compared with the cosmological evolution timescale. We can thus replace $\rho(t)$ by $\rho_{0}$, which is taken to be the density of the CBR photons when the observer jumps into the $\mathrm{BH}$. In what follows we take $\rho_{0}$ to be the present density of the CBR photons. We thus obtain $\dot{n}(\tau)=\rho_{0} \sigma_{\text {cap }} S /\left(4 \pi r_{-}^{2}\right)\left|\kappa_{-} \tau\right|^{-1}$. The total number of incident photons is obtained by integrating $\dot{n}$ over $\tau$ from some $\tau_{\mathrm{c}}$, which is taken, e.g., to be the proper time corresponding to the threshold energy for pair production, to some final proper time $\tau_{\mathrm{f}}$ - which will be taken later to be the proper time at which incident photons are blueshifted to Planck energy. The total number of photons above the threshold energy hitting the infalling object is thus

$$
n\left(\tau_{\mathrm{f}}\right)=\sigma_{\mathrm{cap}} \rho_{0} \kappa_{-}^{-1} S /\left(4 \pi r_{-}^{2}\right) \ln \tau_{\mathrm{c}} / \tau_{\mathrm{f}} .
$$

\section{THE NUMBER OF INTERACTING PHOTONS}

In this section we first discuss the number of the pairproduction events and then we discuss the number of Compton scattering and photo-electric effect events. We shall see that the former is larger than the latter by a factor $Z, Z$ being the electric charge of the nucleus. (However, the contributions of the latter to the thermal effect we shall discuss in Section IV are of the same order of magnitude - see below.) 


\section{A. The Number of Pair-Production Events}

To obtain the actual number of photons interacting with the atomic nuclei or electrons of the infalling object, we need to multiply Eq. (11) by $1-\exp \left(-x \rho_{\mathrm{ob}} \sigma_{e^{+} e^{-}}\right)$, where $\rho_{\mathrm{ob}}$ is the number density of atomic nuclei (electrons), $\sigma_{e^{+} e^{-}}$is the corresponding cross section for pair production, and $x$ is the length of the object in the radial direction (or its thickness). (We use here the constancy of the cross section in the ultra-relativistic limit - see below.) Taking $x \rho_{\mathrm{ob}} \sigma_{e^{+} e^{-}} \ll 1$, we find that the total number of pair-production events per unit volume is

$$
\mathcal{N}_{e^{+} e^{-}}\left(\tau_{\mathrm{f}}\right)=\sigma_{\mathrm{cap}} \sigma_{e^{+} e^{-}} \rho_{0} \rho_{\mathrm{ob}} \kappa_{-}^{-1} /\left(4 \pi r_{-}^{2}\right) \ln \tau_{\mathrm{c}} / \tau_{\mathrm{f}} .
$$

(In fact, our results are still valid even if we relax this assumption, as long as $x \rho_{\mathrm{ob}} \sigma_{e^{+} e^{-}}$is not much larger than unity.) If the infalling object is much smaller than the typical mean free path of the photons, we can assess the interaction strength by calculating the probability that no pair-creation events will occur. It turns out that the majority of the interactions are those which occur in the Coulomb field of the atomic nuclei.

To evaluate the probability that up to $\tau_{\mathrm{f}}$ none of the atomic nuclei (electrons) will interact with the incident photons, we note that from Eq. (2), the number of interactions per nucleus (electron) is

$$
\mathcal{P}=\sigma_{\text {cap }} \sigma_{e^{+} e^{-}} \rho_{0} \kappa_{-}^{-1} /\left(4 \pi r_{-}^{2}\right) \ln \tau_{\mathrm{c}} / \tau_{\mathrm{f}} .
$$

(As we shall see below, $\mathcal{P} \ll 1$.) Therefore, the object will most likely arrive at the $\mathrm{CH}$ without experiencing even a single interaction if the number $\nu$ of its nucleons (electrons) is small compared to $\mathcal{P}^{-1}$.

We express $\sigma_{\text {cap }}$ in terms of the $\mathrm{BH}$ parameters, and find that $\sigma_{\text {cap }}=\pi l M^{2}$, where $l$ is a known dimensionless function of the charge-to-mass ratio of the $\mathrm{BH} q$ [15]. We also find that $\tau_{\mathrm{c}, \mathrm{f}} \approx-\kappa_{-}^{-1} \mathcal{E}_{0} / \mathcal{E}_{\mathrm{c}, \mathrm{f}}$, where $\mathcal{E}_{0}$ is the photon's energy away from the $\mathrm{BH}$.

We now assume that $q=0.998$ [16, and obtain $\sigma_{\text {cap }} \approx 3.5 \times 10^{11}\left(M / M_{\odot}\right)^{2} \mathrm{~cm}^{2}, 1 / r_{-}^{2} \approx$ $5.2 \times 10^{-11}\left(M / M_{\odot}\right)^{-2} \mathrm{~cm}^{-2}$, and $1 / \kappa_{-} \approx 1.0 \times$ $10^{6}\left(M / M_{\odot}\right) \mathrm{cm} . \quad \tau_{\mathrm{c}} \approx-5.0 \times 10^{-15}\left(M / M_{\odot}\right)$ sec and $\tau_{\mathrm{f}}=\tau\left(\mathcal{E}_{\text {Planck }}\right) \approx-6.4 \times 10^{-37}\left(M / M_{\odot}\right)$ sec. Here, $M_{\odot}$ denotes the solar mass.

The Bethe-Heitler formula [17] for the ultra-relativistic cross section for pair production in the Coulomb field of a light nucleus yields, in the case of complete screening, $\sigma_{e^{+} e^{-}}=\alpha r_{\mathrm{c}}^{2} Z^{2}\left[\frac{28}{9} \ln \left(183 Z^{-\frac{1}{3}}\right)-\frac{2}{27}\right]$, where $\alpha$ is the fine-structure constant, and $r_{\mathrm{c}}$ is the classical radius of the electron. (For the corresponding expression for the field of the atomic electrons set $Z=1$.)

We find that $\sigma_{e^{+} e^{-}} \approx 9.4 \times 10^{-27} Z^{2} \mathrm{~cm}^{2}$. Hence, we find that $\mathcal{P} \approx 3 \times 10^{-16}\left(M / M_{\odot}\right) Z^{2}$. Taking $M / M_{\odot}=$ $10^{6}$, we obtain $\mathcal{P} \approx 3 \times 10^{-10} Z^{2}$.

Typically, the number of interactions will be dominated by the Coulomb field of the nuclei and not by the field of the electrons, because the cross section for the former is proportional to $Z^{2}$, while the number of electrons per nuclei is $Z$. Consequently, the total number of events in the field of the nuclei is larger than the number of events in the field of the atomic electrons by a factor $Z$. We conclude that if the object is microscopic, with number of atoms $\nu<\mathcal{P}^{-1} \approx 3 \times 10^{9} Z^{-2}$, it is most likely that none of the atomic nuclei (or electrons) will interact with the incident photons.

A larger object will be affected by the interaction. Yet, we find that only a small fraction-namely, just $3 \times 10^{-10} Z^{2}$ - of its atomic nuclei will interact. However, such a large object might be heated by the energy transfer to the electrons.

\section{B. The Number of Compton and Photo-electric Events}

Ultra-relativistic photons can interact with matter also through ionization of the matter due to the photo-electric effect and the Compton effect. In the limit of extremely hard photons, we expect these two effects to behave similarly. Consequently, we shall discuss in detail only the latter.

The cross section for the Compton effect in the ultra-relativistic limit is given by 18 . $\sigma_{\mathrm{Com}}(\mathcal{E})=$ $\pi r_{\mathrm{c}}^{2} \frac{\mathcal{E}_{\mathrm{c}}}{\mathcal{E}}\left(\ln \frac{2 \mathcal{E}}{\mathcal{E}_{\mathrm{c}}}+\frac{1}{2}\right)$. The total number of events will be given by integrating the product of this cross section and the flux of the incident photons over the proper time up to the $\mathrm{CH}$. One finds, then, that the total number of Compton scatterings per atomic electron is

$$
\mathcal{N}_{\mathrm{Com}}=-\mathcal{N}_{0} \int_{1}^{\infty} \frac{\mathcal{E}_{\mathrm{c}}^{2}}{\mathcal{E}^{2}}\left(\ln \frac{2 \mathcal{E}}{\mathcal{E}_{\mathrm{c}}}+\frac{1}{2}\right) d\left(\frac{\mathcal{E}}{\mathcal{E}_{\mathrm{c}}}\right)
$$

where $\mathcal{N}_{0}=\rho_{0} \sigma_{\text {cap }} /\left(4 \kappa_{-}\right)\left(r_{\mathrm{c}} / r_{-}\right)^{2}$. Here, the lower limit of the integration is taken to be the energy of the incident photon corresponding to the electron mass $m$. For the same numerical values as above, one finds that $\mathcal{N}_{\text {Com }} \approx 3 \times 10^{-16}\left(M / M_{\odot}\right)$. This is of the same order as the number of pair-production events in the field of the atomic electrons. (Recall that the total number of pairproduction events in the field of the nuclei is larger by a factor $Z$.) Note that $\mathcal{N}_{\text {Com }}$ is dominated by the lower limit of the integral: the number of events at higher energies is vanishing with the increase in the energy. Therefore, we assume that all the Compton scattering events occur at energies of the same order as $m$.

\section{THE HEATING EFFECT}

In this section we first calculate the thermal effects due to the pair-creation processes, which are dominated by the atomic electrons, and then we discuss the thermal effects due to Compton scattering. 
The energy of each interacting photon is extremely large, and is growing on the approach to the $\mathrm{CH}$ up to (and possibly even beyond) Planckian levels. Hence, if the size of the object in the radial direction is much larger than the typical mean free path for the relevant processes, one would expect that a considerable portion of the energy of the photons will be absorbed by the object. As this energy is very large, the inevitable result is the complete destruction of the object. This expectation is based on the scenario of a multiplicative shower, in which incident photons produce $e^{+}-e^{-}$pairs, which, in turn, produce even more photons through Bremsstrahlung processes. These photons are expected to create more pairs, etc. As the typical mean free paths for pair production and Bremsstrahlung in the ultra-relativistic limit are roughly equal (for water they are each of order $50 \mathrm{~cm}$ ), one could simplify the calculations by considering the effects of a single interaction for which the effective mean free path is $\lambda$. (The effects of other processes, e.g., annihilation of the created positrons with atomic electrons, are expected to be negligible.) For a thick object we find that the result we shall obtain below in Eq. (5) should be multiplied by a factor $e^{x / \lambda}$. Hence, for $x$ of the same order as $\lambda$, one would obtain just a numerical factor of order $1-10$.

However, if the object is thin compared to $\lambda$, the created pair will most probably cross the object and leave it without any further interaction. Consequently, we shall discuss objects of typical thickness smaller than $\lambda$. (Note that we do not assume $x \ll \lambda$.) As the created pair does not interact with the object, the only source of energy which can be absorbed by the later is the recoil of the nuclei or of the electrons during the interaction. Due to exchange effects the maximum momentum transfer in the pair production process (in the field of the atomic electrons or nuclei) is of order $m$ [19,20]. As the momentum of the photon is much larger than $m$, its momentum is almost completely transferred to the created pair, which will consequently move almost in the same direction of the original photon.

The absorbed energy is inversely proportional to the mass of the nucleus or the electron. Consequently, the energy absorbed by the recoiling electrons is much larger than the energy absorbed by the nuclei. Therefore, the energy absorption by the nuclei is smaller by a factor $Z m / \mu$, where $\mu$ is the mass of the nucleus. We shall focus, therefore, on the thermal effects due to the interaction with the electrons. We shall make the simplifying assumption, that the energy absorption is dominated by the large momentum transfer (LMT) events. Namely, we shall consider here only the events in which the momentum transfer is of order $m$. However, we then assume that in all the events the momentum transfer is $m$. (This is just an order-of-magnitude estimate. Therefore, there is an unknown factor of order unity in our results.)

The LMT cross-section is $\sigma_{e^{+} e^{-}}^{\operatorname{lmt}}=(82 / 27) \alpha r_{\mathrm{c}}^{2}$ 21, 19. We then assume that all of the transferred energy is absorbed in the infalling body as thermal energy, which is manifested by the heating of the object. (The stopping range in biological matter for electrons with kinetic energy of order $0.25 \mathrm{MeV}$ is less than $1 \mathrm{~mm}$ 22. Hence, if our object were such that $x \geq 1 \mathrm{~mm}$ we indeed find that all of the kinetic energy would be absorbed through all channels of energy loss by charged particles moving in matter.)

We thus find that the thermal energy absorbed by the infalling object up to Planck energy is

$$
K \approx(\sqrt{2}-1) m c^{2} \sigma_{\mathrm{cap}} \sigma_{e^{+} e^{-}}^{\operatorname{lmt}} \rho_{0} \kappa_{-}^{-1} /\left(4 \pi r_{-}^{2}\right) \ln \tau_{\mathrm{c}} / \tau_{\mathrm{f}} .
$$

Taking the numerical values for a $\mathrm{BH}$ with $M=10^{6} M_{\odot}$ and $q=0.998$ we find that the absorbed thermal energy per gramme is $k \approx 0.6 \mathrm{~J} / \mathrm{g}$, where we took water to be the matter the infalling object is made of. (It turns out that biological matter - as it is composed mainly of waterhave very similar physical properties to water. Therefore, we simplify our calculations by modelling the infalling object to be made of liquid water.) This corresponds to an increase in the temperature of $0.1 \mathrm{~K}$.

The contribution of Compton scattering to the thermal effects is expected to be of the same order as the contribution of the pair-production events calculated above: Recall that the number of Compton scatterings is of the same order as the number of pair productions in the field of the atomic electrons. As the former is dominated by incident energies of order $m$, we approximate the energy transferred to the ejected electron by $m$. Consequently, a repetition of the above analysis yields for the contribution to the increase in the temperature again $0.1 \mathrm{~K}$.

Note that we did not consider the contribution of interactions a lower energies. Indeed, the threshold energy for pair production sets a lower cutoff in the energy of the incident photon. However, there is no corresponding cutoff when Compton scattering or the photoelectric effect are concerned. Therefore, a more complete analysis of the effects experienced by the infalling object should include these effects also at energies corresponding to the non-relativistic limit up to $m$. In addition, it turns out that the effects caused by the excitation and the photodisintegration of the nuclei are also not expected to be catastrophic. Consequently, we expect that the overall interaction of the infalling object with the CBR photons is bounded and small.

We have found that the more massive the $\mathrm{BH}$, the stronger the interaction of the blue-shifted CBR photons with the infalling object. Therefore, to decrease the extent of this interaction, it would be natural to take less massive BHs. However, in such BHs tidal effects could be disastrous for physical objects.

In conclusion, we find that the thermal heating of the infalling object could be small enough to allow us not to exclude the possibility that it will arrive negligibly damaged at the $\mathrm{CH}$. However, note that as far as a living observer is concerned, this heating corresponds to radioactive radiation of $0.1 \mathrm{~K}$. It should be remembered, 
that such a radiation would probably be fatal for a macroscopic living organism such as a human being. However, micro-organisms may arrive at the $\mathrm{CH}$ without experiencing even a single interaction, and for them the thermal effects would consequently be irrelevant. A non-living object whose typical size is $1-10 \mathrm{~cm}$ or smaller might also be destroyed by this radiation unless it is insensitive to such radioactive radiation. Yet, this investigation does not provide support to the idea that the $\mathrm{CH}$ is to be regarded as a wall which cannot be traversed (or as the boundary of spacetime).

\section{ACKNOWLEDGEMENTS}

I would like to thank Aharon Bar-David, Joshua Feinberg, Yithak Gertner, and Norma Sánchez. It is especially a great pleasure for me to thank Amos Ori for many helpful and clarifying discussions.

[1] E. Poisson and W. Israel, Phys. Rev. D 41, 1796 (1990).

[2] R. Penrose, in Battelle Rencontres, 1967 Lectures in Mathematics and Physics, edited by C. DeWitt and J.A. Wheeler (W.A. Benjamin, New-York, 1968), p. 222.

[3] A. Ori, Phys. Rev. Lett. 67, 789 (1991).

[4] A. Ori, Phys. Rev. Lett. 68, 2117 (1992).

[5] See, however, an outline of a calculation scheme for such perturbations in A. Ori, Perturbative approach to the inner structure of a rotating black hole, submitted.

[6] R.H. Price, Phys. Rev. D 5, 2419 (1972).

[7] C. Gundlach, R.H. Price and J. Pullin, Phys. Rev. D 49, 883 (1994); 49890 (1994); L.M. Burko and A. Ori, in preparation.

[8] M. Simpson and R. Penrose, Int. J. Theor. Phys. 7, 183 (1973); J.M. McNamara, Proc. R. Soc. London A 358, 499 (1978); 364, 121 (1978); Y. Gürsel, V.D. Sandberg, I.D. Novikov and A.A. Starobinsky, Phys. Rev. D 19, 413 (1979).

[9] S. Chandrasekhar and J.B. Hartle, Proc. R. Soc. London A 384, 301 (1982).

[10] L.M. Burko and A. Ori, Phys. Rev. Lett. 74, 1064 (1995).

[11] R. Balbinot, C. Barrabès and A. Fabbri, Phys. Rev. D 49, 2801 (1994).

[12] I.D. Novikov and A.A. Starobinsky, Zh. Eksp. Teor. Fiz. 78, 3 (1980) [Sov. Phys. - JETP 51, 1 (1980)].

[13] D. Marković and E. Poisson, Phys. Rev. Lett. 74, 1280 (1995).

[14] R. Balbinot, C. Barrabès and A. Fabbri, Phys. Rev. D 51, 2782 (1995).

[15] A.F. Zakharov, Class. Quan. Grav. 11, 1027 (1994).

[16] K.S. Thorne, Astrophys. J. 191, 507 (1974).

[17] H. Bethe and W. Heitler, Proc. R. Soc. London A 146, 83 (1934).
[18] W. Heitler, The Quantum Theory of Radiation, third edition (Oxford University Press, London, 1954).

[19] J. Joseph and F. Rohrlich, Rev. Mod. Phys. 30, 354 (1958).

[20] V.B. Berestetskii, E.M. Lifshitz and L.P. Pitaevskii, Quantum Electrodynamics, Landau and Lifshitz Course of Theoretical Physics Volume 4, second edition (Pergamon Press, Oxford, 1982).

[21] J.A. Wheeler and W.E. Lamb, Jr., Phys. Rev. 55, 858 (1939).

[22] Stopping Power for Electrons and Positrons, ICRU Report No. 37, 1 October 1984, Bethesda, MD. (1984). 